\title{
Modern treatments for severe depression still have poor long term outcome
}

Kennedy N, Abbott R, Paykel ES. Remission and recurrence of depression in the maintenance era:

long-term outcome in a Cambridge cohort. Psychol Med 2003;33:827-38.

\section{Has the long term prognosis of people with severe depression changed as a result of the increased use of modern treatments such as antidepressants and psychological therapy?}

METHODS

$-$

Design: Long term follow up of observational study

Setting: Local treatment centres in Cambridgeshire, UK recruitment 1990 to 1992.

으 Population: 70 people diagnosed as having moderate to severe primary unipolar major depression according to the Research Diagnostic Criteria (RDC). Exclusions: concurrent major physical or psychiatric illnesses. Participants had been recruited as part of a 30 month short term follow up study. 69 (99\%) of the original sample were followed up.

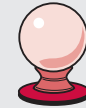

Prognostic factors: Participants were contacted 8 to 11 years after recruitment. A psychiatrist blinded to the original study results reviewed their psychiatric and general practice case notes. The psychiatrist interviewed the participants using an adapted Longitudinal Interview Follow-up Evaluation (LIFE) to assess the number and length of depressive episodes during follow up.

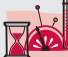

Outcomes: Recurrence of depression (as diagnosed by RDC)

Follow up period: 8-11 years.

\section{MAIN RESULTS}

Eighty five per cent of participants had taken antidepressants for at least one year during follow up, while over $75 \%$ received at least one course of psychological treatment. $92 \%$ of participants (excludes 4 deaths and 1 loss to follow up) recovered from their index depressive episode (ie at least 2 consecutive months with less than 3 RDC symptoms). Of the recovered participants, $67 \%$ had a recurrence of depression during follow up.

\section{CONCLUSIONS}

Despite modern treatments that are highly effective at treating severe depression in the short term, long term recurrence rates remain high.

For correspondence: Dr N Kennedy, Department of Psychiatry, University of Cambridge, Addenbrooke's Hospital, Cambridge, UK

Source of funding: supported by a grant from the University of Cambridge, Department of Psychiatry.

\section{NOTES}

Authors note that participants were heterogeneous in terms of number of previous depressive episodes and course of illness, thus results cannot easily be generalised. In addition they note that a single interview was used to cover a long follow up period, which would have resulted in recall bias.

\section{Commentary}

his is an 8-11 year naturalistic, cohort study of 70 people with depression. The majority were treated as inpatients at index presentation and followed up in primary care and through psychiatric services. Despite a good recovery rate and evidence of access to psychological therapies and maintenance drug treatment, the great majority experienced recurrence during the follow up period.

The authors point out that high recurrence rates have not changed in the last twenty years despite advances in treatment. These findings are commensurate with those of other recent studies in which only $12 \%$ of depressed patients recovered and remained continuously well after 25 years of follow up. ${ }^{1}$ This rather pessimistic long term outcome is compounded by the exclusion of people with major physical illness which is acknowledged as having a significant and deleterious effect on outcome in terms of prognosis over the first year of follow up. ${ }^{2}$ Notably increased age and greater severity of index episode predicted slower recovery. Severity of index episode and number of previous episodes were associated with early recurrence.

However, these pessimistic findings should not mask some of the radical improvements that have taken place in the acute management of depression even in the context of severe physical illness. ${ }^{3}$ Relatively few people suffered from chronic episodes of depression during the follow up period. It is evident that people with depression were able to hold jobs, had good levels of social functioning, and were able to maintain stable relationships. There is no doubt there is considerable room for improvement in our long term management of depression, but it is through aggressive treatment and comprehensive follow up that we can mitigate the adversity experienced by these individuals.

Professor Kenneth Wilson

University of Liverpool, Liverpool, UK

1 Brodaty H, Luscombe G, Peisah C, et al. A 25-year longitudinal, comparison study of the outcome of depression. Psychol Med 2001;31:1347-59.

2 Murphy E. The prognosis of depression in old age. Br J Psychiatry 1983:142:111-19.

3 Gill D, Hatcher S. Antidepressants for depression in medical illness. The Cochrane library, 2003, Issue 3 Update Software. 